\title{
Impact of bacterial chaperonin GroEL-GroES on bacteriorhodopsin folding and membrane integration
}

\author{
Xinwei Lu' ${ }^{1}$, Baomei $\mathrm{Xu}^{1}$, Haiyan Sun ${ }^{1}$, Junting $\mathrm{Wei}^{1}$, Haixia $\mathrm{Chi}^{1}$, \\ Naseer Ullah Khan ${ }^{1}$, Xiaojuan Wang ${ }^{1}$, Xiaoqiang Wang ${ }^{1 \bowtie}$, Fang Huang ${ }^{1 \bowtie}$ \\ 1 State Key Laboratory of Heavy Oil Processing, College of Chemical Engineering, China University of Petroleum \\ (East China), Qingdao 266580, China
}

Received: 13 November 2018 / Accepted: 23 January 2019 / Published online: 11 July 2019

\begin{abstract}
Our understanding of molecular chaperone function in membrane protein biogenesis lags far behind that in soluble protein biogenesis. Through a combined approach including isothermal titration calorimetry, UV-Vis spectroscopy, and fluorescence spectroscopy, the behavior of ATP-dependent chaperonin GroEL-GroES, a paradigmatic chaperone of soluble protein folding, was investigated in the refolding of membrane protein bacteriorhodopsin (BR) and its membrane insertion. We found that BR bound asymmetrically to the double-ring GroEL, with a much higher affinity when it was partially denatured. GroEL alone showed a clear influence on BR refolding, but the presence of ATP was necessary to significantly enhance both the rate and yield of the GroEL-mediated folding, in contrast to the adverse effect of GroES on the folding yield. However, synergy between ATP and GroES was shown to be required not only for releasing high-affinity BR species from GroEL, but also for unfolding and rescuing the misfolded conformers complexed to GroEL. This is consistent with the observation that maximum rate enhancement of BR refolding or assembly with the prepared inverted membrane vesicles was achieved when the complete chaperonin system was used. Our results support the iterative unfolding mechanism of GroEL activity previously proposed for soluble proteins, whereby GroEL might perform repeated unfolding and release of $\mathrm{BR}$, thus offering additional opportunities for timely folding or membrane integration. This work provides important information on the convergence of folding of membrane and soluble proteins in light of folding pathways and the role of molecular chaperones.
\end{abstract}

Keywords Chaperonin, Membrane protein, Membrane reconstitution, Protein folding, Protein structure

\section{INTRODUCTION}

Newly translated proteins are assisted by an integrated network of molecular chaperones in facilitating their efficient folding, post-translational targeting, or even degradation (Castanie-Cornet et al. 2014; Hartl et al. 2011; Kim et al. 2013; Kriegenburg et al. 2012). General

Electronic supplementary material The online version of this article (https://doi.org/10.1007/s41048-019-0090-6) contains supplementary material, which is available to authorized users.

$\triangle$ Correspondence: wangxq001@upc.edu.cn (X. Wang), fhuang@upc.edu.cn (F. Huang) principles of chaperone-mediated protein processing have emerged from extensive studies with water-soluble proteins, but it is unclear whether or how these concepts can be translated into membrane proteins, while the notion that protein structure is determined by amino-acid sequences is well accepted (Anfinsen 1973). Membrane proteins reside in the lipid bilayer and perform numerous biological processes from signaling to transport (Almen et al. 2009; Sharpe et al. 2010; Stevens et al. 2004). However, in the past two decades, biochemical, genetic, and structural studies have revealed both the presence and identities of abundant chaperones involved in virtually all aspects of membrane protein biogenesis, including 
translation, targeting, translocation, and integration into biological membranes (Rapoport et al. 2004; Schnell and Hebert 2003; White and von Heijne 2004).

The chaperonins stand out among ATP-dependent chaperones in that they constitute large megadalton double-ring complexes with a central cavity in each ring (Lin and Rye 2006). Apart from their recognized function in soluble protein folding, chaperonins are also able to modulate membrane protein folding, expression, or the membrane integration. For example, the eukaryotic TRiC/CCT chaperonin complex has been shown to be highly involved in regulating aggregation of nascent GPCR membrane proteins and in promoting their proper maturation and expression (Genier et al. 2016). Meanwhile, it was observed that the chaperonin in chloroplasts can prevent incorrect membrane insertion in cooperation with the cpSec1 machine, and chaperonin-dependent protein unfolding was speculated to play an important role in this process (Endow et al. 2015). GroEL of Escherichia coli has also been shown to interact with SecA, a central component of the translocation machinery (Bochkareva et al. 1998), and facilitate post-translational membrane insertion of the membrane protein lactose permease (Bochkareva et al. 1996). These studies have demonstrated the important roles of chaperonins from all three kingdoms of life in securing the proper biogenesis of membrane proteins.

The bacterial GroEL-GroES chaperonin system is the best characterized molecular chaperone. The essential GroEL is a large barrel-shaped protein complex composed of two heptameric rings of $57 \mathrm{kDa}$ subunits stacked back-to-back, while the lid-shaped co-factor GroES consists of $10 \mathrm{kDa}$ subunits arranged in a domeshaped heptameric ring (Fig. 1A-C) (Hayer-Hartl et al. 2016). The apical domains of GroEL form the entrance to the ring cavity, serving to bind non-native substrate proteins and GroES mainly via hydrophobic interaction. We have found recently that the folding of newly translated polypeptide chain of CCR5 or CXCR4 membrane protein was significantly enhanced by GroELGroES in a cell-free system, as were their structural stability and functional expression (Chi et al. 2015, 2016). These observations provide direct evidence for GroEL-assisted membrane protein folding, but the underlying mechanisms remain to be elucidated. In the GroEL-mediated folding of soluble proteins, both passive and active models have been proposed, which differ in whether GroEL-GroES solely acts passively by preventing aggregation or actively modifies a substrate protein's structure or accessible conformational space (Hayer-Hartl et al. 2016). In either case, the assisted folding leads to the burial of non-polar amino-acid residues within the interior of soluble proteins. By contrast, whether folded or not, membrane proteins have abundant hydrophobic surface residues. This raises the question how would GroEL-GroES act in a dramatically different folding reaction to form the hydrophobic, native protein?

Herein, this question is addressed by using bacteriorhodopsin (BR) as a model integral membrane protein, which comprises seven transmembrane $\alpha$-helices connected by short extramembrane loops (Fig. 1D). Refolding of BR from a denatured state can be readily analyzed by recovery of the characteristic adsorption band of the correctly bound retinal chromophore (Booth 1997). We directly examined the binding mode of both denatured and native BR to apoGroEL, and then studied the effects on BR refolding and the protein structure of GroEL, or combined with ATP or/and GroES. The non-ionic detergent n-dodecyl- $\beta$-D-maltoside was used as a solubilizing reagent in the refolding process. In addition, we also show how the chaperonin system modulates the membrane integration of either denatured or native BR. Our study is not only beneficial to deepening our understanding of the convergence between chaperoning membrane proteins and chaperoning soluble proteins, but also helpful for a full interpretation of mechanism of GroEL function in folding or even membrane integration, since there is no characteristic difference between membrane proteins and authentic GroEL-dependent proteins that are typically highly aggregation prone (Calloni et al. 2012; Fujiwara et al. 2010; Kerner et al. 2005).

\section{RESULTS}

\section{Both denatured and native BR can bind to GroEL}

Central to the mechanism of GroEL activity is its ability to recognize a diverse range of polypeptides mainly through interactions between the hydrophobic residues of the substrate and helices $\mathrm{H}$ and I on the GroEL apical domains (Fig. 1A) (Coyle et al. 1997). Here, binding of both denatured and native BR to GroEL was first examined by isothermal titration calorimetry (ITC), since each state has abundant hydrophobic surface residues that are readily accessible to GroEL. As shown in Fig. 2A, titration of GroEL with BR yields exothermic titration thermograms in both cases. The heat changes are due specifically to binding of BR to GroEL as consecutive injection of BR into the assay buffers without the chaperonin produces flat thermograms (supplemental Fig. S1). Heat of each injection shown in Fig. 2A was integrated, corrected by the dilution heat, and was plotted against the molar ratio of BR: GroEL (Fig. 2B). 

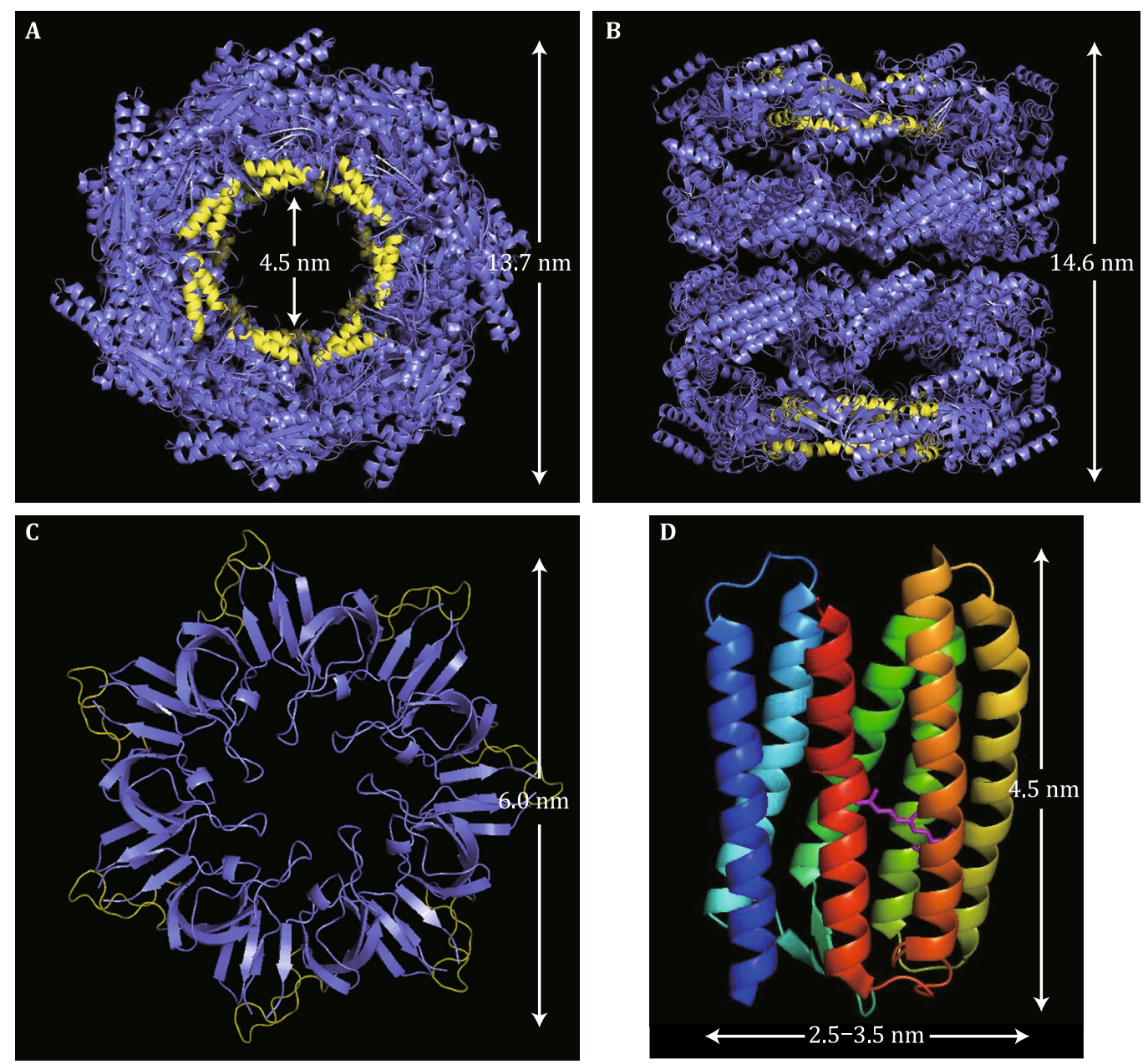

Fig. 1 Three-dimensional structures of GroEL, GroES, and BR used in this study. A Top view of GroEL showing helices H and I (yellow) facing the central cavity. B Side view of GroEL showing the two heptameric rings that are stacked back-to-back (PDB ID: 1SS8). C Top view of GroES showing the mobile loops (dark yellow), through which GroES binds to GroEL (PDB ID: 1PF9). D Side view of BR showing seven transmembrane helices with a retinal chromophore (pink) bound to a lysine side group (PDB ID: 1IW6). The structures of GroEL, GroES, and BR are rendered at different scales as indicated

The heat change fits one set of sites binding model (red and blue curves), yielding a dissociation constant $\left(K_{\mathrm{d}}\right)$ near $0.3 \mathrm{nmol} / \mathrm{L}$ for denatured BR and near $6.0 \mathrm{nmol} / \mathrm{L}$ for native BR, respectively (Table 1). Thus the denaturation of BR increased the binding affinity of GroEL for this membrane protein by one order of magnitude. On the other hand, binding of either BR sample is driven by favorable enthalpy change $(\Delta H)$ and opposed by negative entropy change $(\Delta S)$; the binding stoichiometry $(N)$ determined in both cases is close to unity (Table 1). However, binding of the native BR is clearly less enthalpic with less entropy compensation.

The lid-shaped co-chaperonin GroES is an essential component in GroEL-mediated protein folding in bacteria, and has been shown to share the same binding sites on GroEL with the substrate (Chen and Sigler 1999). Titration of GroEL with denatured BR in the presence of GroES indicates that GroES has little effect on BR binding (supplemental Fig. S2 and Table 1). This result can be understood by taking $K_{\mathrm{d}}$ into account, which for the formation of a GroEL/ES complex was previously determined to be $3 \mu \mathrm{mol} / \mathrm{L}$ and is significantly higher than that of BR-GroEL complex determined here (Behlke et al. 1997). This indicates a much weaker interaction of the two chaperonin proteins and hence consistent with the insignificant effect. However, in the presence of ATP, which regulates the binding and release cycles of GroEL and GroES in vivo, the affinity of GroEL/ES would increase three orders of magnitude (normally $K_{\mathrm{d}} \sim 1 \mathrm{nmol} / \mathrm{L}$ ) (Farr et al. 2000), theoretically capable of competing with BR binding to GroEL. Effect of apoGroEL on BR folding and then the role of GroES and ATP in this process were subsequently investigated. 

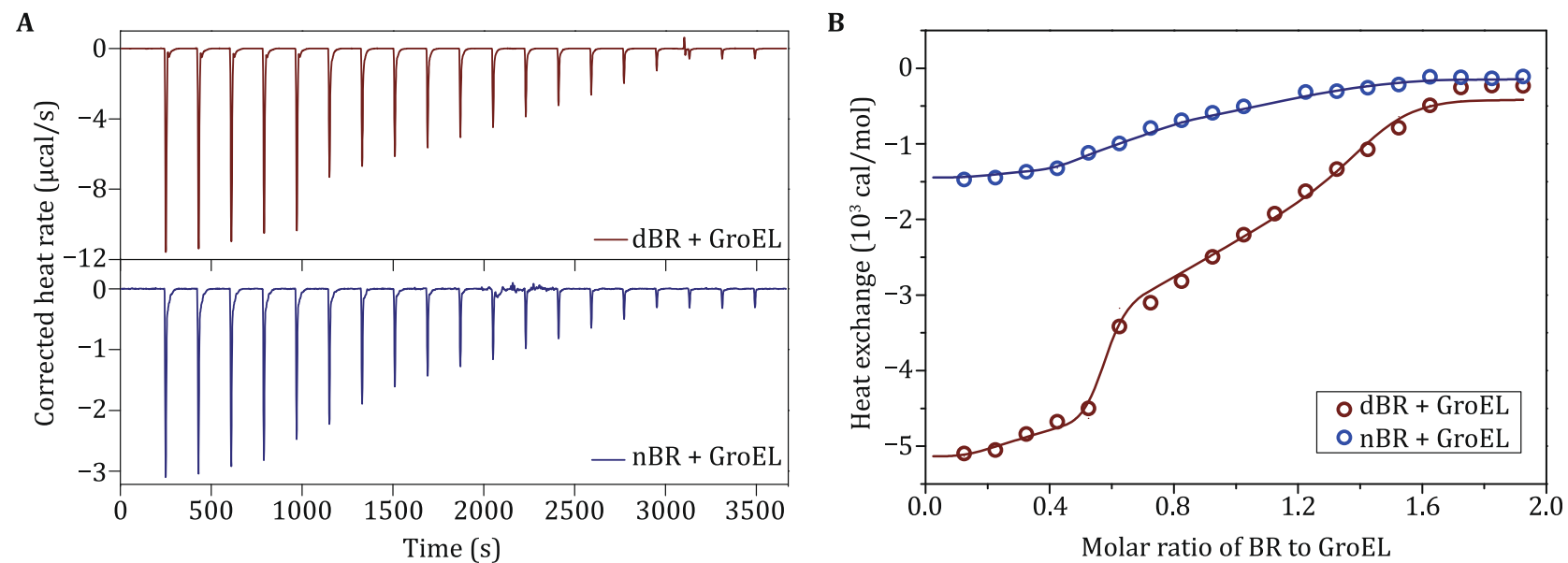

Fig. 2 BR binding to GroEL assessed by ITC. A Titration of GroEL with SDS-denatured BR (dBR, red) or native BR (nBR. blue). Thermograms were recorded at $20{ }^{\circ} \mathrm{C}$ with a MicroCal ITC200 instrument. B Heat exchange of each injection in A was integrated and plotted vs the BR:GroEL molar ratio. Solid lines represent the fit of data to a single set of sites model (all sites identical and equivalent) and the thermodynamic parameters obtained are listed in Table 1

Table 1 Thermodynamic parameters of BR binding to apoGroEL or in the presence of GroES

\begin{tabular}{|c|c|c|c|c|c|}
\hline Titrant (Syringe) & Titrand (Cell) & $K_{\mathrm{d}}(\mathrm{nmol} / \mathrm{L})$ & $\Delta H(\mathrm{cal} / \mathrm{mol})$ & $\Delta S(\mathrm{cal} /(\mathrm{mol} \cdot \mathrm{K}))$ & $N$ \\
\hline $\mathrm{dBR}$ & GroEL & $0.3 \pm 0.1$ & $-5135.2 \pm 55.3$ & $-17.5 \pm 1.6$ & $0.87 \pm 0.01$ \\
\hline nBR & GroEL & $6.0 \pm 1.3$ & $-1455.6 \pm 34.2$ & $-4.9 \pm 0.4$ & $0.88 \pm 0.03$ \\
\hline $\mathrm{dBR}$ & GroEL/ES & $0.5 \pm 0.2$ & $-5086.5 \pm 57.5$ & $-17.3 \pm 0.9$ & $0.86 \pm 0.01$ \\
\hline
\end{tabular}

\section{GroEL-GroES system can modulate BR folding in the presence of DDM}

A portion of SDS-denatured BR was observed to refold when diluted with an excess of solubilizing detergent $\mathrm{n}$-dodecyl- $\beta$-D-maltoside (DDM), as revealed by measuring the recovery of retinal absorption (supplemental Fig. S3) (Booth 1997). No significant recovery was detectable in the assay buffer without detergent or supplemented with GroEL alone. However, in the presence of DDM, the addition of GroEL showed an obvious effect on BR folding (supplemental Fig. S3). Rate constant for the GroEL $(0.15 \mu \mathrm{mol} / \mathrm{L})$-mediated folding, as assessed by fitting with single exponential kinetics, was estimated to be $\sim$ twofold lower than the spontaneous folding. GroEL is known to typically retard the folding of soluble proteins that can fold efficiently in its absence. This has been explained through a competition between intramolecular folding and intermolecular binding to GroEL (Gray and Fersht 1993; Itzhaki et al. 1995). It seems entirely plausible that GroEL behaves similarly in the refolding of denatured $\mathrm{BR}$. When the concentration of GroEL was increased to $0.30 \mu \mathrm{mol} / \mathrm{L}$, the estimated rate constant did not change obviously, whereas the yield of folding reached a much higher level than the spontaneous folding after $60 \mathrm{~min}$ (supplemental Fig. S3). These data demonstrate that apoGroEL can decrease BR folding rate but enhance the yield of the folded protein.

Because bacterial cells contain several millimolars of ATP and under normal conditions, the relative molar ratio of GroES versus GroEL is 1.9 and after heat shock 4.7 (Moparthi et al. 2013); GroEL will unlikely stay long without binding ATP and GroES. In the presence of ATP (blue in Fig. 3A), the recovery of correctly folded BR was significantly faster and greater compared to that with apoGroEL alone or the spontaneous process (red and black in Fig. 3A). In contrast, the combination of GroEL and GroES showed little effect on the rate of spontaneous folding but reduced the yield to some extent (Fig. 3A, cyan). This indicates an interaction between GroEL and GroES with no requirement of ATP, probably induced by BR bound to GroEL, which in turn had an adverse effect on the recovery of correctly folded protein. When the complete chaperonin system was used (Fig. 3A, green), maximum rate enhancement was observed but the folding yield fell in between the two preceding cases.

The above results indicate that ATP and GroES can influence the GroEL-mediated BR folding in a different 
A

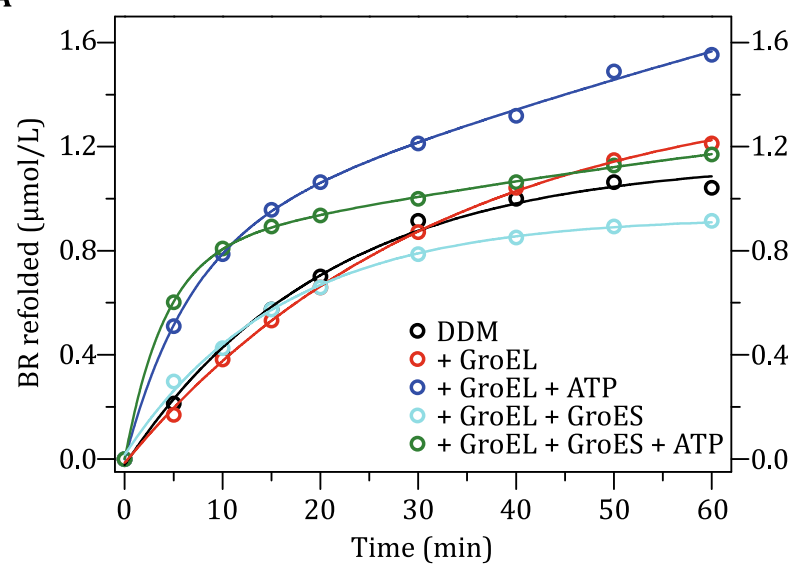

C

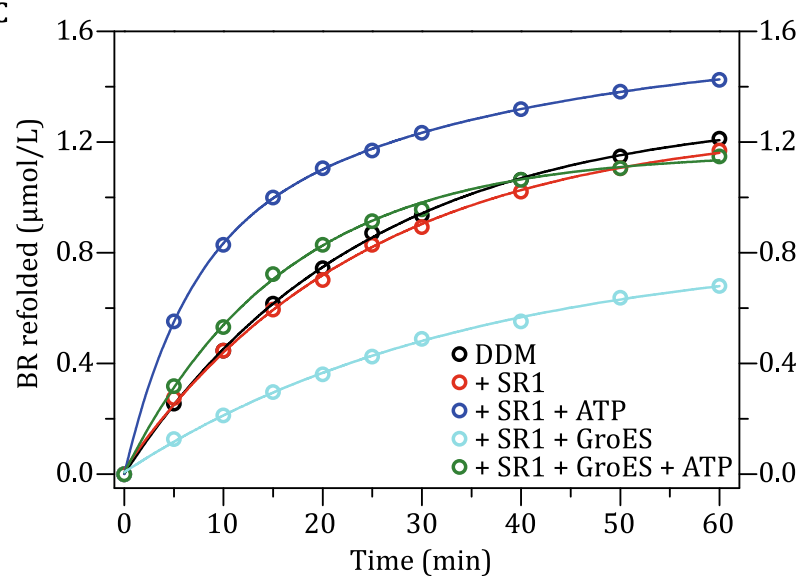

B

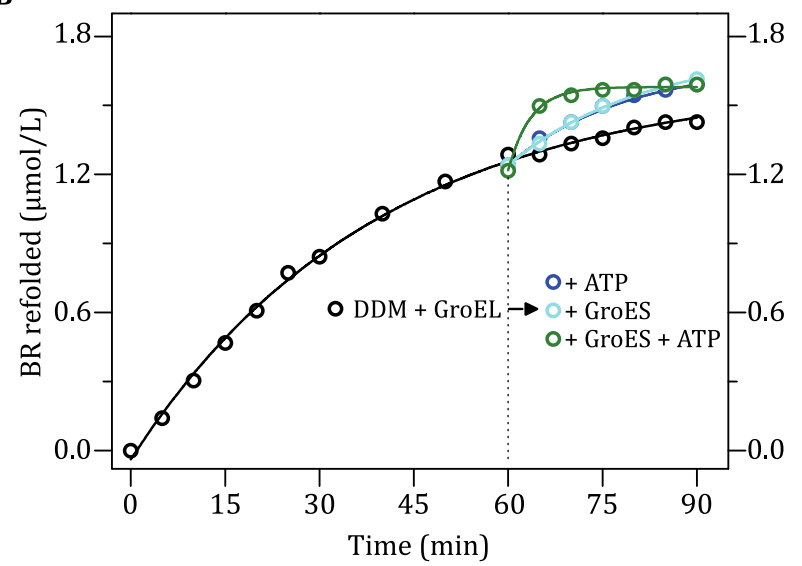

D

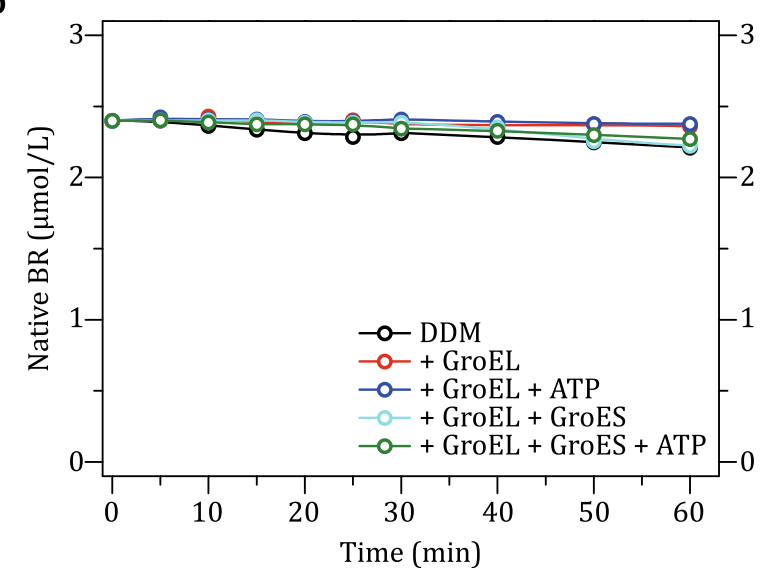

Fig. 3 Time course of BR folding modulated by ATP-dependent GroEL-GroES system. A The recovery of folded BR was continuously monitored with absorbance at $554 \mathrm{~nm}$. The following additions were made: none (black); $0.3 \mu \mathrm{mol} / \mathrm{L}$ GroEL (red); $0.3 \mu \mathrm{mol} / \mathrm{L}$ GroEL and $5 \mathrm{mmol} / \mathrm{L}$ ATP (blue); $0.3 \mu \mathrm{mol} / \mathrm{L}$ GroEL and $0.6 \mu \mathrm{mol} / \mathrm{L}$ GroES (cyan); $0.3 \mu \mathrm{mol} / \mathrm{L}$ GroEL, $0.6 \mu \mathrm{mol} / \mathrm{L}$ GroES and $5 \mathrm{mmol} / \mathrm{L}$ ATP (green). B Net GroEL-mediated BR folding was carried out first; then at $T=60$ min the sample was supplemented with only ATP, with only GroES, or with both as indicated. C For comparison with A, the effect of non-cycling single-ring (SR1) version of GroEL on BR folding was also analyzed. The concentrations of SR1, GroES, and ATP used were $0.6 \mu \mathrm{mol} / \mathrm{L}, 1.2 \mu \mathrm{mol} / \mathrm{L}$, and $5 \mathrm{mmol} / \mathrm{L}$, respectively. D The effects of GroEL/ES plus ATP on the native fold of BR were examined by following the absorbance change at $560 \mathrm{~nm}$. The concentrations of chaperonin and nucleotide used in $\mathbf{B}$ and $\mathbf{D}$ were the same as in A. BR was kept at $2.4 \mu \mathrm{mol} / \mathrm{L}$ in all experiments. The blue and green lines in $\mathbf{A}$ and the blue line in $\mathbf{C}$ represent the fit of data to three-phase exponential equation, while the remaining lines are the fit with single exponential equation. All the lines in $\mathbf{B}$ and $\mathbf{D}$ are simple guides for the eyes

manner. GroES binding to GroEL is somewhat detrimental to the folding, in contrast to the well-known positive role of substrate encapsulation in the GroEL/ES cage for the assisted folding (Jewett and Shea 2010). We next questioned how this adverse effect was produced. Intriguingly, GroES alone or its mobile loop sequence through which GroES binds to GroEL also facilitated the recovery of folded BR (supplemental Fig. S4). Moreover, the loop sequence showed an effect similar to GroES in the GroEL-mediated folding in the absence and presence of ATP (supplemental Fig. S4). Although it cannot form a sealed cap above the GroEL central cavity like GroES, implying a significant contribution from the loop-GroEL interaction to the folding. Notably, the hydrophobic residues at the GroEL's apical surface involved in binding GroES mobile loop mostly overlap with those involved in binding the substrate protein (Motojima et al. 2000). It is unlikely that the hydrophobic BR was displaced and freed into the hydrophilic GroEL/ES cage by the mobile loop, especially given the presence of DDM micelles outside of the cage, which would further favor the folding or solubilization of BR. Preferably, as demonstrated for the assisted folding of several soluble proteins (Motojima and Yoshida 2010), BR may be captured near the GroEL/ES interface, partly protruding to outside when the whole system or even GroEL/ES alone was used in this study. Such an interaction may prevent BR from accessing the favorable DDM micelles, thus resulting in the reduced folding yield observed with both GroES and its mobile loop. 
However, some studies suggest that the role of ATP and GroES is only to dissociate sticky substrates (i.e., with a $K_{\mathrm{d}}$ in the nmol/L region determined here), which limit the assisted folding rate or even inhibit the folding activity of GroEL if not removed (Priya et al. 2013). To test whether GroES and ATP show a similar effect in assisted BR folding, we first incubated apoGroEL with denatured BR for $60 \mathrm{~min}$; then, we added GroEL or/and ATP (Fig. 3B). The subsequent addition of either component further promoted BR folding, but was less effective than the simultaneous addition of both, demonstrating synergism between the two at helping the GroEL-mediated folding. Anisotropy measurements with BR transmembrane peptides, which were used as mimetics for denatured BR to circumvent the complications introduced by the dynamic nature of denatured BR and of GroEL-BR interactions, showed that only the combination of ATP and GroES was capable of efficiently separating the preformed peptide-GroEL complex (supplemental Fig. S5). Thus, it seems that both GroES and ATP are necessary to dissociate high-affinity BR conformers and regenerate the stalled GroEL binding (or catalytic) sites.

To further examine the effect of GroES and ATP on the GroEL-mediated BR folding, we used a single-ring GroEL mutant (SR1) that forms a stable complex with GroES (Weissman et al. 1995), in contrast to the cycling GroEL-GroES system. Similar to our observation with GroEL, ATP increased the rate and the yield of SR1mediated folding (blue in Fig. 3C), while the presence of GroES reduced both aspects considerably (cyan) when compared to the case with apoSR1 alone or the spontaneous process (red or black). As denatured BR was determined by ITC to bind to SR1 with a stoichiometry near unity (supplemental Fig. S2), and SR1 used was twice the concentration of GroEL, we speculate that more BR-SR1 complexes formed than BR-GroEL, whereby GroES exerted more influence on the folding (cyan). As expected, addition of both ATP and GroES showed an insignificant effect on BR recovery (green), ascribable to the irreversible binding of GroES to SR1 in the presence of ATP (Weissman et al. 1995). Moreover, this result indicates that the maximum rate enhancement observed with GroEL is due to ATP-regulated multiple cycles of binding and releasing of GroES.

GroEL alone or in combination with ATP or/and GroES showed negligible influence on the structure of native BR solubilized with DDM (Fig. 3D), pointing to a stronger intramolecular interaction within the native protein than intermolecular binding to GroEL. Thus the chaperonin-mediated transition of BR from the metastable denatured state to the native state is thermodynamically favorable.

\section{Evidence for both the chaperonin-mediated disaggregation and unfolding}

Measuring the spontaneous BR folding at varied concentrations demonstrates that aggregation resulted in only partial recovery of correctly folded BR and the apparent rate was concentration independent, implying that aggregation was irreversible (supplemental Fig. S6). In the absence of solubilizing detergent DDM, SDS-denatured BR was estimated by fluorescence correlation spectroscopy (FCS) to have a diffusion coefficient $\left(\sim 67 \mu \mathrm{m}^{2} / \mathrm{s}\right)$ much lower than bound to GroEL $\left(\sim 104 \mu \mathrm{m}^{2} / \mathrm{s}\right.$ ) (Fig. 4). This reflects that the aggregates formed by the denatured BR are even larger than the BR-GroEL complex with a binding stoichiometry near unity as determined by ITC. This also means that GroEL was able to disrupt the aggregation structure, essentially pumping the productive folding pathway with monomeric BR. Moreover, the nBR solubilized with DDM was determined to have a diffusion coefficient of $\sim 117 \mu \mathrm{m}^{2} / \mathrm{s}$, which is greater than that of $\mathrm{dBR}$ with GroEL and much greater than that of dBR alone (Fig. 4). This suggests that $\mathrm{nBR}$ was well dispersed in the presence of DDM and also supports the idea of GroELmediated disaggregation of dBR. Besides, when the complete chaperonin system was added into denatured $\mathrm{BR}$, white flocculent precipitates were immediately visible (data not shown), indicating forced unfolding as well as synergism between GroES and ATP in this aspect. Without solubilizing detergents or lipids that mimic biological membranes in the bulk solution,

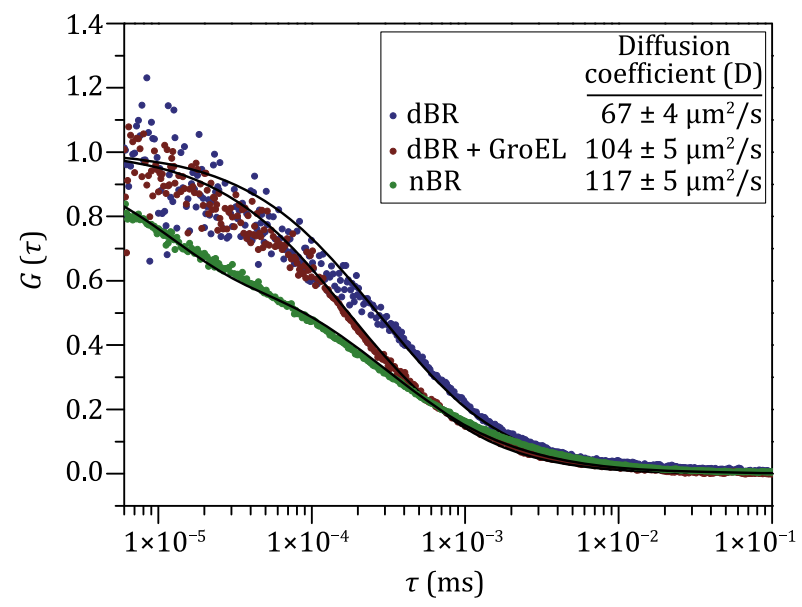

Fig. 4 FCS measurement of denatured BR in the absence or presence of GroEL compared to native BR. Fluorescence autocorrelation amplitudes $G(\tau)$ of Alexa Fluor 488 fluorescence was shown for SDS-denatured BR alone or with an excess of GroEL in absence of solubilizing detergent or native BR solubilized with DDM. Diffusion coefficients (D) were obtained by fitting the autocorrelation curve with Eq. 1. Standard deviations were from three independent measurements 
unfolded BR would instantly aggregate and precipitate, in contrast to the significant folding rate increase observed in the presence of DDM.

\section{Membrane insertion of BR mediated by GroEL- GroES}

To determine whether or how GroEL-GroES would support integration of BR into the bilayer, inverted cytoplasmic membrane vesicles (IMVs) were prepared from Escherichia. coli cells and mixed with denatured BR in the presence and absence of apoGroEL, or plus ATP/ GroES (Fig. 5A). ApoGroEL caused an insignificant change in the recovery of correctly folded BR in IMVs, as measured by UV-Vis spectroscopy (black and red). Different from the refolding in DDM micelles, addition of GroEL and ATP was shown to be detrimental to the membrane insertion (blue), while GroEL combined with GroES facilitated this process (cyan). The real reason for this reproducible difference is not known, but the rigidity of IMVs and the structural change of GroELbound BR induced by ATP or GroES binding might be possible reasons. Specifically, ATP binding by GroEL is known to cause an expansion of the opening to the cavity of the chaperonin (Skjaerven et al. 2015). This change is more appreciable than that caused by merely GroES binding to GroEL (Kim et al. 2005), thus possibly enabling the unfolding of the bound substrate (Lin et al. 2008; Sharma et al. 2008), favorable to productive folding in an appropriate solvent. However, unlike DDM micelles that is highly dynamic, IMVs was probably unable to promptly protect the unfolded BR species and provide timely an advantageous folding microenvironment. In comparison, the weak association of GroES with GroEL in the absence of ATP might deliver BR to the prepared IMVs in a more efficient manner. However, similar to the refolding in DDM micelles, the complete GroEL-GroES system greatly enhanced the membrane insertion of BR (green), which reached a steady state quickly with the amount of recovered BR falling in between the two preceding cases.

Next, fluorescence anisotropy was employed to probe the effects of the bacterial chaperonins on the insertion of BR into IMVs (Fig. 5B). When fluorescently labeled native $\mathrm{BR}$ was mixed with an excessive amount of GroEL, the anisotropy shifted to a higher value, demonstrating that the membrane protein formed a stable complex with the chaperonin. Importantly, the subsequent addition of IMVs led to a further increase of anisotropy, indicative of the integration of BR into IMVs. ATP and GroES were found to further enhance the transfer of BR into the membrane, as judged also by the anisotropy increase. These results raise the prospect that GroEL, along with GroES and ATP, may have a direct role in the integration of proteins into the lipid bilayer in vivo.

\section{DISCUSSION}

Forces of GroEL-protein binding are exerted at the GroEL-protein interfaces. In principle, titration calorimetry is reflecting energy changes within the entire system rather than reporting only the GroELprotein binding. The energy changes observed here can be considered to be the sum of energy changes derived
A

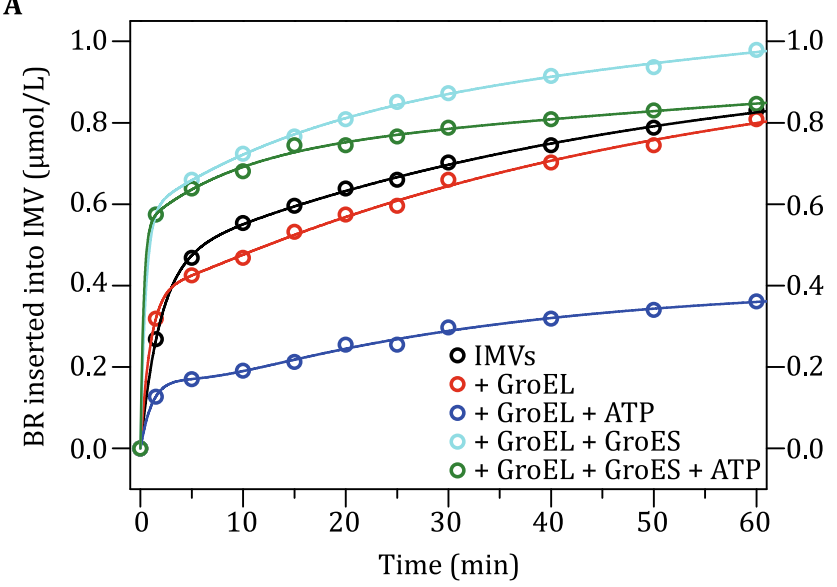

B

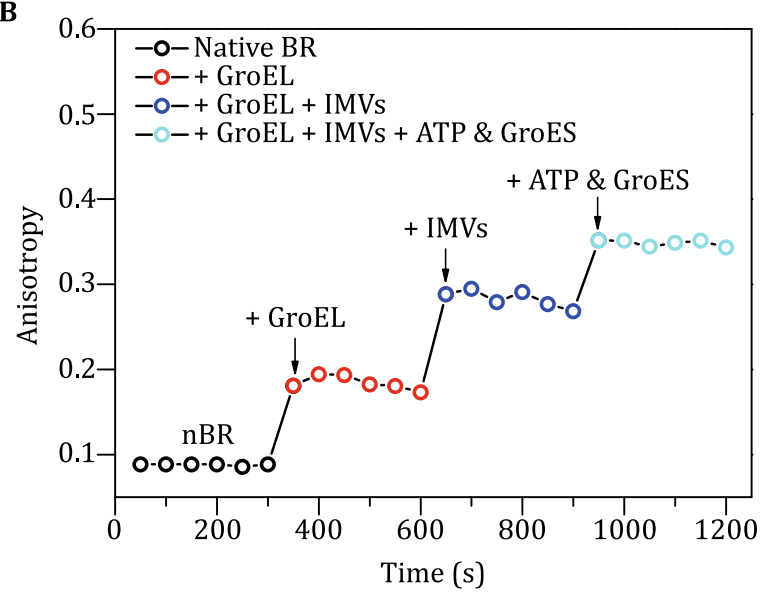

Fig. 5 Insertion of BR into IMVs mediated by the GroEL-GroES system. A Insertion and/or refolding of denatured BR (2.4 $\mu$ mol/L) in IMVs were continuously monitored with absorbance at $554 \mathrm{~nm}$. The following additions were made: none (black); $0.3 \mu \mathrm{mol} / \mathrm{L}$ GroEL (red); $0.3 \mu \mathrm{mol} / \mathrm{L}$ GroEL and $5 \mathrm{mmol} / \mathrm{L}$ ATP (blue); $0.3 \mu \mathrm{mol} / \mathrm{L}$ GroEL and $0.6 \mu \mathrm{mol} / \mathrm{L}$ GroES (cyan); $0.3 \mu \mathrm{mol} / \mathrm{L} \mathrm{GroEL,} 0.6 \mu \mathrm{mol} / \mathrm{L}$ GroES and $5 \mathrm{mmol} / \mathrm{L}$ ATP (green). B Native BR can be efficiently transferred to IMVs in the presence of GroEL with the help of ATP and GroES. The tested concentrations of native BR, GroEL, GroES, and ATP were 0.4, 5, $10 \mu \mathrm{mol} / \mathrm{L}$, and $5 \mathrm{mmol} / \mathrm{L}$, respectively 
from GroEL-BR binding and those from the displacement of lipids and detergents that originally surrounded the BR hydrophobic segment. Titration calorimetry can provide information on the thermodynamic properties of binding reaction itself only if the contribution of the latter fractions is little. It is conceivable that the lipids and detergents displaced from GroEL-BR interfaces into bulk solution will bind again to other fractions through hydrophobic effect, so that the overall energy change derived from the displacement should not be significant. Thus, it is reasonable to assume that the energy changes observed here are primarily caused from the interaction of GroEL with BR.

The most common distinguishing feature of GroEL substrates is the presence of exposed hydrophobic surface area. From this standpoint, there is no distinguishable boundary between non-native soluble proteins exposing considerable hydrophobic residues and denatured membrane proteins. However, in order to truly drive the transition of a membrane protein from the denatured state to the native state, GroEL must be able to discriminate them, i.e., preferentially interacting with the denatured state, which are both hydrophobic. Here, we have shown that the affinity of BR for GroEL is enhanced about tenfold due to SDS denaturation, although the native conformer itself also displays a high affinity. This is likely due to their different binding valences with a GroEL ring of seven apical binding sites. Multivalent binding by GroEL has been shown for several non-native soluble proteins (Farr et al. 2000; Saibil 2013). In comparison with native BR comprising a tightly assembled bundle of helices, its SDS-denatured state has very loosely packed amino acids and residual structure (Krishnamani et al. 2012), thus presumably allowing GroEL to bind with the entire protein molecule through more sites (valences) and to show a higher affinity and biased substrate selectivity during the mediated folding. It is conceivable that, to facilitate folding, evolutionary pressures give rise to seven nonconsecutive binding sites in each GroEL ring that ensure not only efficient binding via hydrophobic interactions, but also substrate discrimination via structural compactness. This may also be one reason that GroEL can efficiently promote the folding of newly translated membrane proteins (Chi et al. 2015, 2016) for which the lengthy structure would engage more binding sites than the folded state and thus have competitive advantage in binding to GroEL.

Despite the fact that GroEL consists of two back-toback stacked homoheptameric rings, our results demonstrate that only one BR, whether denatured or not, binds to GroEL, leaving one GroEL ring unoccupied with the protein. Similarly, native BR and two other membrane proteins (LacY and the bacteriophage $\lambda$ holin) have been shown to bind asymmetrically to GroEL using single-particle analysis of negatively stained protein-GroEL complexes, after the dialysis of solubilizing detergent (Sun et al. 2005). This binding mode is in agreement with the prevalent view that the two GroEL rings work alternatively like a two-stroke engine in assisting the folding of soluble proteins, with only one ring active at any given time (Horwich et al. 2006). The negative inter-ring allostery of GroEL in binding BR or substrate proteins in general indicates that effect of the bound protein is transmitted across the ring and alters the protein binding function of the open trans ring, similar to the well-known ATP-induced negative inter-ring cooperativity (Gruber and Horovitz 2016), although the underlying mechanism at the molecular level remains still to be determined.

In the kinetic studies of BR folding, apoGroEL shows two opposite effects: decreasing the rate but increasing the recovery of folded protein in the presence of DDM. These effects may result from an entirely passive process. For example, the interaction of non-native BR with GroEL competes with the intramolecular folding, thus slowing the folding rate, while it would also favorably alter kinetic partitioning. By maintaining a lower level of free-folding intermediates, aggregation is reduced, leading to more of the protein folding into its native state (Lin and Rye 2006). This possibility is strongly supported by the ability of GroEL to block the aggregation of SDS-denatured BR, as revealed by FCS measurements. On the other hand, it has been demonstrated that apoGroEL possesses intrinsic unfoldase activity that is mediated by the hydrophobic interaction of the folding intermediate with the channel surface of the GroEL apical domains (Libich et al. 2015). It is tempting to speculate that apoGroEL may additionally unfold the off-pathway BR conformers after intermolecular binding, and subsequently release them into DDM micelles where further attempts at correct folding can occur.

Our results demonstrate that while the combination of GroEL and ATP can significantly increase both the rate and yield of BR refolding, the combination of GroEL and GroES has an adverse effect on the yield. This is reasonable when taking into account the presence of DDM micelles in bulk solution that would be beneficial to BR refolding and solubilization. Cooperative binding of ATP to the seven subunits of a GroEL ring is known to induce an upward and clockwise movement of the apical domains responsible for substrate binding (Skjaerven et al. 2015). Such a conformational change may release weakly bound BR segments, while mediating an expansion of tightly bound segments, closely followed by further release and refolding of BR that can readily 
occur in the surrounding DDM micelles. Despite the fact of negating the functional significance of in-cage confinement, this mode of action can rescue misfolded BR states kinetically stabilized by non-native contacts and has been proposed previously for GroEL-aided folding of soluble proteins (Lin et al. 2008; Lin and Rye 2006; Sharma et al. 2008). By contrast, the formation of GroEL/ES complex even without ATP provides only an isolated, hydrophilic folding environment. BR intermediates, either trapped completely in the cage or partially at the GroEL/ES interface as speculated in this study, would be discouraged from promptly refolding in a favorable environment (i.e., the surrounding DDM micelles), thus increasing the chance of stepping onto a non-productive pathway. However, the possibility of forced unfolding upon GroES binding to GroEL cannot be ruled out either.

BR refolding with maximum rate has been shown to be achievable in the presence of the complete chaperonin system implying an important contribution from cooperation of ATP and GroES. This is consistent with their notable effects on the structure of denatured BR in the absence of DDM indicative of forced unfolding. Furthermore, the study with non-cycling SR1 suggests that multiple cycles of binding and releasing of GroES are necessary to efficiently accelerate the folding process. Collectively, these results can be interpreted in support of an iterative unfolding/annealing mechanism of GroEL-GroES action (Thirumalai and Lorimer 2001), whereby the essential GroEL with the help of GroES and ATP is capable of timely reversing misfolded, kinetically trapped BR states, affording them a new chance to fold, thus accelerating the folding process. This mechanism and the potential function of ATP and GroES in regenerating GroEL activity, as revealed in this work, are not mutually exclusive and may act in synergy during the mediated BR refolding.

In addition to their roles in BR refolding, GroELGroES can also modulate integration of BR into membranes. The complete chaperonin system appears to be more efficient in promoting the delivery of either denatured or native BR into the bilayer. At a minimum, this points to the significance of molecular chaperones for the productive biogenesis of membrane proteins. However, further investigation is needed to clarify the exact role of GroEL-GroES or how they interact with other components in membrane integration in vivo.

\section{CONCLUSIONS}

In summary, we have studied the impact of the bacterial chaperonin GroEL-GroES on the folding and assembly of
BR with biological membranes and the underlying mechanisms. We first examined the binding of BR to GroEL by ITC, and found that BR, either in a denatured or native state, binds asymmetrically to GroEL, but denaturation can significantly increase the affinity of the chaperonin for BR. This lays the foundation of preferred interaction of GroEL with denatured BR, and explains partially why the chaperonin system can drive the folding reaction. The subsequent folding studies indicate that ATP and GroES have opposite effects on the GroEL-mediated BR refolding. However, cooperation of ATP and GroES was shown to be necessary to maximize the folding rate, and to dissociate the sticky BR species and regenerate GroEL activity. By considering together all our results, we propose that GroEL with the help of ATP and GroES can mediate iterative cycles of binding, unfolding, release, and refolding of BR. In addition, we found that the complete chaperonin system efficiently transfers the denatured or native BR into the bilayer. These results underline the functional flexibility of the fascinating molecular machine of GroEL-GroES. While recognizing that actual membrane protein biogenesis is a very complex process, we believe that the results presented would be instrumental for understanding how cells invest in molecular chaperones to prevent protein aggregation and promote efficient folding and membrane assembly.

\section{EXPERIMENTAL PROCEDURES}

\section{Strains, plasmids, and proteins}

GroEL, its single-ring mutant SR1, and GroES were prepared to $\sim 95 \%$ purity using E. coli strain BL21 harboring the plasmid pTric 99 as previously described (Braig et al. 1994; Molugu et al. 2015). The plasmids carrying the gene of GroEL, SR1, or GroES were kind gifts from Professor Arthur L. Horwich in Yale University. The concentrations of GroEL, SR1, and GroES were estimated by the Bradford method. Native BR or its mutants used were expressed with the shuttle vector PBA2 and the host cell MPK409, and purified thereafter as previously described (Sun 2013). The BR samples thus obtained were in the purple membrane, which have been demonstrated to adopt a native conformation via a combined approach of circular dichroism, UV-Vis spectroscopy, and fluorescence spectroscopy (Sun 2013). The mobile loop peptide from GroES $\left(\mathrm{NH}_{2}-\right.$ KRKEVETKSAGGIVLTGSAA-COOH) and the modified first transmembrane peptide of BR (TAMRAKKIWLALGTALMGLGTLYFLVKK) were synthesized and purified ( $>95 \%$ purity) by Nanjing Impact BioScience 
Ltd. 5(6)-carboxytetramethylrhodamine (TAMRA) was attached to the N-terminus of BR peptide; meanwhile, we added two hydrophilic amino acids $(\mathrm{K})$ at the termini to increase the solubility.

\section{Binding of BR to GroEL assessed by ITC}

The ITC experiments with both denatured BR and native BR were carried out at $20.0{ }^{\circ} \mathrm{C}$ using a MicroCal $^{\mathrm{TM}}$ ITC200 instrument (GE Healthcare). The denatured BR sample was prepared by incubating native purple membrane containing BR in $0.25 \%(w / v)$ SDS for $2 \mathrm{~h}$ at room temperature, and then dilution with HEPES buffer (25 mmol/L HEPES-NaOH, $120 \mathrm{mmol} / \mathrm{L} \mathrm{NaCl}, \mathrm{pH}$ 7.5). The native sample was prepared by dissolving purple membrane directly into HEPES buffer plus n-dodecyl- $\beta$ D-maltoside (DDM, Sigma-Aldrich) with a final concentration of $0.55 \mathrm{mmol} / \mathrm{L}$, which is a mild non-ionic detergent and was used to disrupt the membrane. DDM has a critical micelle concentration (CMC) of around $0.17 \mathrm{mmol} / \mathrm{L}$, and showed no detrimental effect on the native conformation of BR even at a concentration up to $10 \times$ CMC (Sun 2013). GroEL or its single-ring mutant SR1 was prepared by using the same buffer. During ITC measurements, a micro-syringe was used to titrate BR sample (denatured or native; $10 \mu \mathrm{mol} / \mathrm{L}$ ) into the calorimeter cell containing GroEL (or SR1) solution $(1 \mu \mathrm{mol} / \mathrm{L})$. When GroES was used in combination with GroEL, their molar ratio was kept at 2:1. Control experiments were performed by titrating BR into the assay buffer. Thermodynamic parameters were obtained from the titration curve fitted using the one set of sites model of Origin software supplied with the instrument.

\section{GroEL folding assays}

Folding assays were performed by 16-fold dilution (unless otherwise stated) of denatured BR into folding buffer (25 mmol/L HEPES (pH 7.5), $20 \mathrm{mmol} / \mathrm{L} \mathrm{KCl}$, $10 \mathrm{mmol} / \mathrm{L} \mathrm{MgCl}_{2}$ ) in the presence or absence of the detergent DDM (7.8 mmol/L) with different chaperonin and ATP concentrations, as mentioned in the figure legends. The denatured BR was prepared as described above. Folding was followed by monitoring the evolution of the absorbance at $554 \mathrm{~nm}$ in a 1-mL cuvette with constant stirring (Dahmane et al. 2013). When the mobile loop peptide from GroES was used in combination with GroEL, their molar ratio was kept at 14:1.

\section{Isolation of IMVs}

The "percoll gradient methodology" following Morein et al. IMVs was used for preparation of inverted cytoplasmic membrane vesicles (IMVs) (Morein et al. 1994). Briefly, E. coli BL21 cells were disrupted by passing the cell suspension through a French pressure cell twice, followed by centrifugation at $8000 \mathrm{~g}$ for $30 \mathrm{~min}$ at $4{ }^{\circ} \mathrm{C}$. The supernatant was collected and further centrifuged at $100,000 \mathrm{~g}$ for $1 \mathrm{~h}$ at $4{ }^{\circ} \mathrm{C}$. The membrane pellet obtained was then suspended in $25 \mathrm{mmol} / \mathrm{L}$ phosphate buffer (pH 7.5) containing $0.2 \mathrm{mmol} / \mathrm{L}$ EDTA, $1 \mathrm{mmol} / \mathrm{L}$ DTT, and $16.2 \%(\mathrm{v} / \mathrm{v})$ Percoll. IMVs were isolated by centrifuging the membrane-Percoll mixture at 27,000 $\mathrm{g}$ in a P70AT-870 rotor at $4{ }^{\circ} \mathrm{C}$ with a Hitachi himac Cp80WX ultracentrifuge for $30 \mathrm{~min}$. A very faint low-density band close to the top of the Percoll gradient consists predominantly of vesicles derived from the cytoplasmic membrane. The inner membrane-bound enzyme NADH-oxidase was used a marker and the activity measurement supported that the low-density membrane fraction obtained was IMVs (supplemental Fig. S7). This fraction was carefully collected and then centrifuged at $265,000 \mathrm{~g}$ to remove the Percoll from the sample. GroEL-mediated BR folding in the presence of IMVs was carried out as described above. The IMVs used for BR folding or membrane insertion were determined to have a diameter of around $200 \mathrm{~nm}$, as assessed by dynamic light scattering (data not shown), and an enzymatic activity of 7.9 units $/ \mathrm{mL}$.

\section{Anisotropy measurements}

The synthesized BR transmembrane peptide was first dissolved in a small amount of hexafluoroisopropanol, and then diluted well into HEPES buffer $(25 \mathrm{mmol} / \mathrm{L}$ HEPES ( $\mathrm{pH}$ 7.5)]. Effects of GroES/ATP on the interaction of GroEL with the TAMRA-tagged BR peptide were monitored by steady-state anisotropy on a FluoroMax-4 instrument (Horiba). The excitation and emission wavelengths for TAMRA were $560 \mathrm{~nm}$ (slit width $5 \mathrm{~nm}$ ) and $582 \mathrm{~nm}$ (slit width $5 \mathrm{~nm}$ ), respectively. A BR 0CV130C mutant was prepared and labeled with Alexa Fluor 488. The effects of bacterial chaperonins on the insertion of the fluorescent BR mono-dispersed with DDM into IMVs were also examined by anisotropy. The excitation wavelength was $488 \mathrm{~nm}$ (slit width $5 \mathrm{~nm}$ ) with an emission wavelength at $514 \mathrm{~nm}$ (slit width $5 \mathrm{~nm}$ ). The addition of GroEL, ATP or/and GroES, or IMVs, and their final concentrations were as indicated in the figures or the legends.

\section{Fluorescence correlation spectroscopy}

FCS measurements were performed with a BR V130C mutant labeled with Alexa Fluor 488 on a Nikon Eclipse Ti-U inverted microscope, which was maintained at a 
constant temperature of $20{ }^{\circ} \mathrm{C}$. For excitation of Alexa 488 , the laser at $488 \mathrm{~nm}$ was used. The excitation light was guided through an oil immersion objective $(100 \times 1.49 \mathrm{NA}$, Olympus) into the sample cuvette. The auto-correlation function describing three-dimensional Brownian diffusion is expressed as follows (Eq. 1), which was used to fit the auto-correlation curve obtained for SDS-denatured BR $(150 \mathrm{nmol} / \mathrm{L})$ in the absence or presence of GroEL $(1.5 \mu \mathrm{mol} / \mathrm{L})$ to estimate the diffusion coefficient $(D)$.

$G(\tau)=N \cdot \frac{1}{1+\frac{4 D \tau}{\omega_{0}^{2}}} \cdot \frac{1}{\sqrt{1+\frac{4 D \tau}{z_{0}^{2}}}}$,

where $G(\tau)$ and $\tau$ represent fluorescence autocorrelation amplitude and diffusion time; $N$ is the average number of fluorescent molecules in the detection volume; $\omega_{0}$ and $z_{0}$ are the lateral and axial radii, respectively, whose ratio defines the shape of the detection volume.

Acknowledgements This work was supported by the National Natural Science Foundation of China (21503278), China Postdoctoral Science Foundation (2014M560588, 2015T80756), the Fundamental Research Funds for the Central Universities, and the Key Technologies R\&D Program of Shandong Province (2018GGX102025, 2018GSF121039).

\section{Compliance with Ethical Standards}

Conflict of interest Xinwei Lu, Baomei Xu, Haiyan Sun, Junting Wei, Haixia Chi, Naseer Ullah Khan, Xiaojuan Wang, Xiaoqiang Wang, and Fang Huang declare that they have no conflict of interest.

Human and animal rights and informed consent This article does not contain any studies with human or animal subjects performed by any of the authors.

Open Access This article is distributed under the terms of the Creative Commons Attribution 4.0 International License (http:// creativecommons.org/licenses/by/4.0/), which permits unrestricted use, distribution, and reproduction in any medium, provided you give appropriate credit to the original author(s) and the source, provide a link to the Creative Commons license, and indicate if changes were made.

\section{References}

Almen MS, Nordstrom KJV, Fredriksson R, Schioth HB (2009) Mapping the human membrane proteome: a majority of the human membrane proteins can be classified according to function and evolutionary origin. BMC Biol 7:50

Behlke J, Ristau O, Schönfeld HJ (1997) Nucleotide-dependent complex formation between the Escherichia coli chaperonins GroEL and GroES studied under equilibrium conditions. Biochemistry 36:5149-5156
Bochkareva E, Seluanov A, Bibi E, Girshovich A (1996) Chaperonin-promoted post-translational membrane insertion of a multispanning membrane protein lactose permease. J Biol Chem 271:22256-22261

Bochkareva ES, Solovieva ME, Girshovich AS (1998) Targeting of GroEL to SecA on the cytoplasmic membrane of Escherichia coli. Proc Natl Acad Sci USA 95:478-483

Booth PJ (1997) Folding alpha-helical membrane proteins: kinetic studies on bacteriorhodopsin. Fold Des 2:R85-R92

Braig K, Otwinowski Z, Hegde R, Boisvert DC, Joachimiak A, Horwich AL, Sigler PB (1994) The crystal structure of the bacterial chaperonin GroEL at $2.8 \AA$. Nature 371:578-586

Calloni G, Chen T, Schermann SM, Chang HC, Genevaux P, Agostini F, Tartaglia GG, Hayer-Hartl M, Hartl FU (2012) DnaK functions as a central hub in the $E$. coli chaperone network. Cell Rep 1:251-264

Castanie-Cornet MP, Bruel N, Genevaux P (2014) Chaperone networking facilitates protein targeting to the bacterial cytoplasmic membrane. BBA-Mol Cell Res 1843:1442-1456

Chen L, Sigler PB (1999) The crystal structure of a GroEL/peptide complex: plasticity as a basis for substrate diversity. Cell 99:757-768

Chi HX, Wang XQ Li JQ, Ren H, Huang F (2015) Folding of newly translated membrane protein CCR1 is assisted by the chaperonin GroEL-GroES. Sci Rep 5:17037

Chi HX, Wang XQ Li JQ, Ren H, Huang F (2016) Chaperoninenhanced Escherichia coli cell-free expression of functional CXCR11. J Biotechnol 231:193-200

Coyle JE, Jaeger J, Gross M, Robinson CV, Radford SE (1997) Structural and mechanistic consequences of polypeptide binding by GroEL. Fold Des 2:R93-104

Dahmane T, Rappaport F, Popot JL (2013) Amphipol-assisted folding of bacteriorhodopsin in the presence or absence of lipids: functional consequences. Eur Biophys J Biophy 42:85-101

Endow JK, Singhal R, Fernandez DE, Inoue K (2015) Chaperoneassisted post-translational transport of plastidic type I signal peptidase 1. J Biol Chem 290:28778-28791

Farr GW, Furtak K, Rowland MB, Ranson NA, Saibil HR, Kirchhausen T, Horwich AL (2000) Multivalent binding of nonnative substrate proteins by the chaperonin GroEL. Cell 100:561-573

Fujiwara K, Ishihama Y, Nakahigashi K, Soga T, Taguchi H (2010) A systematic survey of in vivo obligate chaperonin-dependent substrates. EMBO J 29:1552-1564

Genier S, Degrandmaison J, Moreau P, Labrecque P, Hebert TE, Parent JL (2016) Regulation of GPCR expression through an interaction with CCT7, a subunit of the CCT/TRiC complex. Mol Biol Cell 27:3800-3812

Gray TE, Fersht AR (1993) Refolding of barnase in the presence of GroE. J Mol Biol 232:1197-1207

Gruber R, Horovitz A (2016) Allosteric mechanisms in chaperonin machines. Chem Rev 116:6588-6606

Hartl FU, Bracher A, Hayer-Hartl M (2011) Molecular chaperones in protein folding and proteostasis. Nature 475:324-332

Hayer-Hartl M, Bracher A, Hartl FU (2016) The GroEL-GroES chaperonin machine: a nano-cage for protein folding. Trends Biochem Sci 41:62-76

Horwich AL, Farr GW, Fenton WA (2006) GroEL-GroES-mediated protein folding. Chem Rev 106:1917-1930

Itzhaki LS, Otzen DE, Fersht AR (1995) Nature and consequences of GroEL-protein interactions. Biochemistry 34:14581-14587

Jewett AI, Shea JE (2010) Reconciling theories of chaperonin accelerated folding with experimental evidence. Cell Mol Life Sci 67:255-276 
Kerner MJ, Naylor DJ, Ishihama Y, Maier T, Chang HC, Stines AP, Georgopoulos C, Frishman D, Hayer-Hartl M, Mann M, Hartl FU (2005) Proteome-wide analysis of chaperonin-dependent protein folding in Escherichia coli. Cell 122:209-220

Kim SY, Semyonov AN, Twieg RJ, Horwich AL, Frydman J, Moerner WE (2005) Probing the sequence of conformationally induced polarity changes in the molecular chaperonin GroEL with fluorescence spectroscopy. J Phys Chem B 109:24517-24525

Kim YE, Hipp MS, Bracher A, Hayer-Hartl M, Hartl FU (2013) Molecular chaperone functions in protein folding and proteostasis. Annu Rev Biochem 82:323-355

Kriegenburg F, Ellgaard L, Hartmann-Petersen R (2012) Molecular chaperones in targeting misfolded proteins for ubiquitindependent degradation. FEBS J 279:532-542

Krishnamani V, Hegde BG, Langen R, Lanyi JK (2012) Secondary and tertiary structure of bacteriorhodopsin in the SDS denatured state. Biochemistry 51:1051-1060

Libich DS, Tugarinov V, Clore GM (2015) Intrinsic unfoldase/foldase activity of the chaperonin GroEL directly demonstrated using multinuclear relaxation-based NMR. Proc Natl Acad Sci USA 112:8817-8823

Lin Z, Rye HS (2006) GroEL-mediated protein folding: making the impossible, possible. Crit Rev Biochem Mol 41:211-239

Lin Z, Madan D, Rye HS (2008) GroEL stimulates protein folding through forced unfolding. Nat Struct Mol Biol 15:303-311

Molugu SK, Li JH, Bernal RA (2015) Separation of E. coli chaperonin groEL from beta-galactosidase without denaturation. J Chromatogr B 1007:93-99

Moparthi SB, Sjölander D, Villebeck L, Jonsson BH, Hammarström P, Carlsson U (2013) Transient conformational remodeling of folding proteins by GroES-individually and in concert with GroEL. J Chem Biol 7:1-15

Morein S, Henricson D, Rilfors L (1994) Separation of inner and outer membrane vesicles from Escherichia coli in selfgenerating Percoll gradients. Anal Biochem 216:47-51

Motojima F, Yoshida M (2010) Polypeptide in the chaperonin cage partly protrudes out and then folds inside or escapes outside. EMBO J 29:4008-4019

Motojima F, Makio T, Aoki K, Makino Y, Kuwajima K, Yoshida M (2000) Hydrophilic residues at the apical domain of GroEL contribute to GroES binding but attenuate polypeptide binding. Biochem Biophys Res Commun 267:842-849

Priya S, Sharma SK, Sood V, Mattoo RUH, Finka A, Azem A, De Los Rios P, Goloubinoff P (2013) GroEL and CCT are catalytic unfoldases mediating out-of-cage polypeptide refolding without ATP. Proc Natl Acad Sci USA 110:7199-7204

Rapoport TA, Goder V, Heinrich SU, Matlack KES (2004) Membrane-protein integration and the role of the translocation channel. Trends Cell Biol 14:568-575

Saibil H (2013) Chaperone machines for protein folding, unfolding and disaggregation. Nat Rev Mol Cell Bio 14:630-642

Schnell DJ, Hebert DN (2003) Protein translocons: multifunctional mediators of protein translocation across membranes. Cell 112:491-505

Sharma S, Chakraborty K, Mueller BK, Astola N, Tang YC, Lamb DC, Hayer-Hartl M, Hartl FU (2008) Monitoring protein conformation along the pathway of chaperonin-assisted folding. Cell 133:142-153

Sharpe HJ, Stevens TJ, Munro S (2010) A Comprehensive comparison of transmembrane domains reveals organellespecific properties. Cell 142:158-169

Skjaerven L, Cuellar J, Martinez A, Valpuesta JM (2015) Dynamics, flexibility, and allostery in molecular chaperonins. FEBS Lett 589:2522-2532

Stevens TJ, Mizuguchi K, Arkin IT (2004) Distinct protein interfaces in transmembrane domains suggest an in vivo folding model. Protein Sci 13:3028-3037

Sun, C. (2013). Folding research of bacteriorhodopsin. Master degree thesis of China University of Petroleum supervised by Prof. Huang, F, 17-21

Sun JC, Savva CG, Deaton J, Kaback HR, Svrakic M, Young R, Holzenburg A (2005) Asymmetric binding of membrane proteins to GroEL. Arch Biochem Biophys 434:352-357

Thirumalai D, Lorimer GH (2001) Chaperonin-mediated protein folding. Annu Rev Bioph Biom 30:245-269

Weissman JS, Hohl CM, Kovalenko O, Kashi Y, Chen S, Braig K, Saibil HR, Fenton WA, Horwich AL (1995) Mechanism of GroEL action: productive release of polypeptide from a sequestered position under GroES. Cell 83:577-587

White SH, von Heijne G (2004) The machinery of membrane protein assembly. Curr Opin Struct Biol 14:397-404 